\title{
Intrinsic magnetic moment on (0001) surfaces of rhombohedral graphite
}

\author{
Minoru Otani, ${ }^{1,4}$ Mikito Koshino, ${ }^{2}$ Yoshiteru Takagi, ${ }^{3,4}$ and Susumu Okada ${ }^{3,4}$ \\ ${ }^{1}$ Research Institute for Computational Sciences, National Institute of Advanced Industrial Science and Technology (AIST), \\ Tsukuba 305-8568, Japan \\ ${ }^{2}$ Department of Physics, Tokyo Institute of Technology, 2-12-1 Oh-okayama, Meguro-ku, Tokyo 152-8551, Japan \\ ${ }^{3}$ Graduate School of Pure and Applied Sciences and Center for Computational Sciences, University of Tsukuba, \\ 1-1-1 Tennodai, Tsukuba 305-8571, Japan \\ ${ }^{4}$ Japan Science and Technology Agency, CREST, 5 Sanbancho, Chiyoda-ku, Tokyo 102-0075, Japan
}

(Received 15 March 2010; published 7 April 2010)

\begin{abstract}
We study electronic structure of the (0001) surfaces of graphite with a rhombohedral stacking arrangement by performing the first-principles total-energy calculations based on the density functional theory. We find that ferrimagnetic spin polarization occurs on the (0001) surfaces of rhombohedral graphite. Detailed analyses of energy bands, spin densities, and wave-function distribution unequivocally reveal the nature of the ferrimagnetic spin polarization, which is associated with the peculiar surface-localized state that possesses the same characteristics as the edge-localized states of graphite flakes.
\end{abstract}

DOI: 10.1103/PhysRevB.81.161403

PACS number(s): 73.22.Pr, 75.70.Rf, 73.20.At

On lattice systems with particular network topology, it is now established that imperfections, such as edges, point defects, and surfaces, lead to an interesting class of electron states around the imperfections. For graphene, whose network topology is characterized by the bipartite lattice, such imperfections result in peculiar electronic states around the Fermi level whose characteristics are completely different from the conventional defect- or surface-induced states. Graphene flakes or ribbons with zigzag edges lead to the emergence of flat-band states in a part of the Brillouin zone at the Fermi energy, which is localized near but extended along the edges. An analytical study showed that flat-band states (i.e., an edge state or border state) result from a delicate balance of electron transfer among the $\pi$ orbitals situated near the edge atoms. ${ }^{1-3}$ Thus, the distribution of the edge state strongly depends on the wave number in a onedimensional Brillouin zone: the edge state is exactly distributed on edge $\mathrm{C}$ sites at the zone boundary of the Brillouin zone. By decreasing the wave number, the edge state loses its localized character and penetrates the inner region of the ribbon. Finally, at $k=2 \pi / 3$, they are smoothly connected to the dispersive bulk $\pi$ and $\pi^{*}$ bands. Thus, the edge state can be classified as a new surface-electron state that is induced by the topological conditions imposed on certain lattice structures. Such edge-localized states near the Fermi level have been observed in a susceptibility measurement experiment and a recent scanning tunneling microscopy experiment. $^{4,5}$

Recent theoretical works have found that the edge state is not peculiar to graphene ribbons with zigzag edges, but occurs at particular edges, interfaces, and surfaces in various lattice structures. ${ }^{6-8}$ Indeed, our previous first-principles total-energy calculations have demonstrated the existence of the surface-localized state on $\mathrm{Si}(111)$ surfaces, whose characteristics are qualitatively the same as those of graphite ribbons with zigzag edges. ${ }^{7}$ In this case, the edge state emerges at a deep energy level $6 \mathrm{eV}$ below the Fermi level because the state originates from the $3 s$ state of the Si atom. Furthermore, analytic calculations based on the single-orbital tightbinding approximation clearly show that the edge state emerges on the surfaces, edges, and interfaces of the various lattice networks that are classified as bipartite lattices. ${ }^{8}$ In this case, the edge state is distributed over one of two sublattices of the bipartite network and exhibits a localized nature in a part of the Brillouin zone. Furthermore, the state exhibits an extended nature in the rest of the Brillouin zone.

In view of the network topology comprising adjacent atomic pairs (not only intralayer but also interlayer pairs), graphite with the rhombohedral $(A B C)$ stacking arrangement is classified as a bipartite lattice. ${ }^{9}$ In fact, the low-energy band of rhombohedral graphite slabs comprises surface states localized on the outermost layers, which form a pair of flat dispersion bands in the vicinity of the Brillouin-zone boundary $K$ and $K^{\prime}$ points just as the edge states in graphene ribbons with zigzag edges. ${ }^{10-13}$ In the present work, we explore the emergence of peculiar surface states of rhombohedral graphite by using numerical calculations based on the density functional theory (DFT) and show that spin polarization occurs in the surface states due to the exchange splitting of the flat dispersion band.

Before discussing the DFT calculations, we derive an analytical expression for the electronic wave functions for the flat-band states within the simplest single-orbital tightbinding model. We consider a semi-infinite rhombohedral graphite slab with a (0001) surface, where $A$ and $B$ on $l$ th layer are labeled as $A_{l}$ and $B_{l}(l=1,2,3, \ldots) . A B C$ stacking has the atomic arrangement shown in Fig. 1, where vertical bonds couple the sites $B_{l}$ and $A_{l+1}$. The primitive translation vectors of single graphene layers are given by $\mathbf{R}_{1}=a(1,0)$ and $\mathbf{R}_{2}=a(-1 / 2, \sqrt{3} / 2)$ with a lattice constant $a=0.246 \mathrm{~nm}$. As the simplest model, we include transfer integrals between intralayer $\left(\gamma_{0}\right)$ and interlayer $\left(\gamma_{1}\right)$ neighboring atoms. Although the atomic structure of rhombohedral graphite is different from common $A B A$-stacked graphite, we expect that the corresponding coupling parameters have similar values. ${ }^{14}$ For typical values of $A B A$ graphite, we quote ${ }^{15} \gamma_{0}$ $=3.16 \mathrm{eV}$ and $\gamma_{1}=0.39 \mathrm{eV}$.

The Schrödinger equation is written as

$$
E \Psi_{A_{l}}=h^{*}(\mathbf{k}) \Psi_{B_{l}}-\gamma_{1} \Psi_{B_{l-1}},
$$


(a)

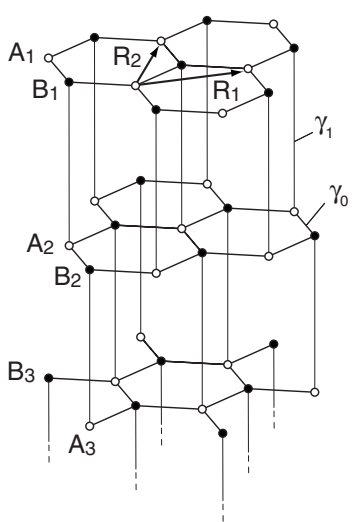

(b)

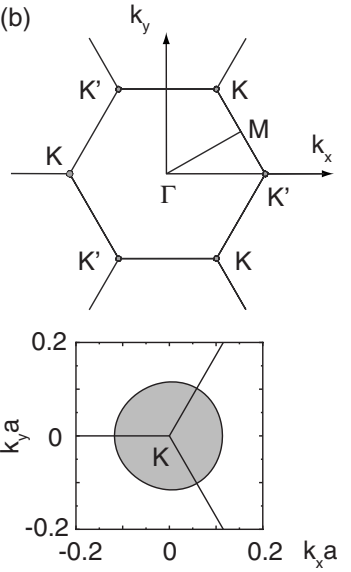

FIG. 1. (a) Reciprocal lattice of rhombohedral graphite with (0001) surfaces. Edge states appear at the shadowed region when $\gamma_{1} / \gamma_{0}=0.1$. (b) Expanded figure around the $K$ point.

$$
E \Psi_{B_{l}}=h(\mathbf{k}) \Psi_{A_{l}}-\gamma_{1} \Psi_{A_{l+1}}
$$

with

$$
h(\mathbf{k})=-\gamma_{0}\left(e^{-i \mathbf{k} \cdot\left(\mathbf{R}_{1}+\mathbf{R}_{2}\right) / 3}+e^{-i \mathbf{k} \cdot\left(-2 \mathbf{R}_{1}+\mathbf{R}_{2}\right) / 3}+e^{-i \mathbf{k} \cdot\left(\mathbf{R}_{1}-2 \mathbf{R}_{2}\right) / 3}\right) .
$$

Here, $\Phi_{X_{l}}(l \geq 1)$ is the wave function on the lattice point $X_{l}$ and $\mathbf{k}=\left(k_{x}, k_{y}\right)$ is the Bloch wave number. We assume that for $l \leq 0, \Phi_{X_{l}}=0$. At zero energy, the tight-binding equation Eq. (2) can be solved as $\Phi_{A_{l}}=\left[h(\mathbf{k}) / \gamma_{1}\right]^{l-1} \Phi_{A_{0}}$ and $\Phi_{B_{l}}=0$, where $\Phi_{A_{0}}$ is the wave function on the $A$ atom of the topmost layer. For the physically relevant solution, we find the convergence condition

$$
\left|h(\mathbf{k}) / \gamma_{1}\right|^{l-1} \leq 1 .
$$

When $\gamma_{1} / \gamma_{0}$ is much smaller than 1 , as expected in a real system, the region of Eq. (3) becomes an almost circular region around the $K$ and $K^{\prime}$ points expressed by

$$
\left|\hbar v \delta k / \gamma_{1}\right|^{l-1} \leq 1
$$

in agreement with the $\mathbf{k} \cdot \mathbf{p}$ approximation, ${ }^{10-13}$ where $\delta k$ is the wave number measured from $K$ or $K^{\prime}$ and $v$ $=\sqrt{3} \gamma_{0} a /(2 \hbar)$ is the velocity of electrons in graphene. Thus, by increasing the interlayer transfer, we expect the flat-band region to increase. Figure 1 shows the region expressed by Eq. (3) with $\gamma_{1} / \gamma_{0}=0.1$, where the circular region is slightly warped in a trigonal manner by the higher-order terms in $\delta k$.

In the DFT calculations, ${ }^{16,17}$ we use the local spin density approximation to express the exchange-correlation energy of interacting electrons. ${ }^{18,19}$ The Vanderbilt ultrasoft pseudopotentials are adopted to describe the electron-ion interaction. ${ }^{20}$ The valence wave functions and charge density are expanded in terms of a plane-wave basis set with a cutoff energy of 25 and 324 Ry, respectively. The surface is simulated using a repeating-slab model in which eight graphene layers and $10 \AA$-vacuum regions are included. For the lateral direction, we use $1 \times 1$ periodicity containing two $\mathrm{C}$ atoms in each layer. To avoid periodic image errors arising from the slab model, ${ }^{21}$ we adopt the effective-screening medium method ${ }^{22}$

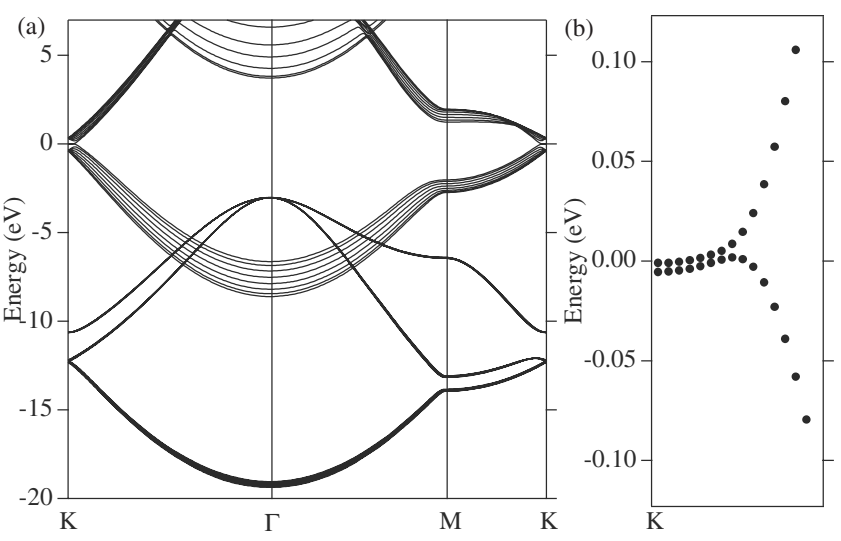

FIG. 2. (a) Electronic energy band of a rhombohedral graphite slab made of eight graphene layers. The energy is measured from the Fermi level. (b) The electronic energy band near the Fermi level in the vicinity of the $K$ point.

in which the Poisson equation is solved by imposing the open-boundary condition normal to the slab. The flat-band region in the two-dimensional Brillouin zone is small, so we have to integrate in the reciprocal lattice on fine meshes to investigate spin polarization in the flat-band states. Thus, to integrate over the surface Brillouin zone, we use equidistant $k$ point sampling of $52 \times 52 k$ points. Geometry optimization has been performed for all atoms in the slab. The conjugategradient minimization scheme has been used both for the electronic structure calculation and for the geometry optimization. In the optimized geometries, the remaining force acting on the atoms is less than $0.005 \mathrm{Ry} / \AA$.

Figure 2 shows the electronic structure of graphite with a rhombohedral stacking arrangement consisting of eight graphene layers. We found that a pair of flat dispersion bands emerged near the Fermi level. The flat-band states keep their flat-band nature around the vicinity of the Brillouin-zone boundary at the $K$ point. The flat-band region is a few percent of the length of $K-\Gamma$ line. The flat-band region on the (0001) surfaces of the rhombohedral graphite is considerably narrower than that of graphene ribbon with zigzag edges. The narrower region is ascribed to the weak interlayer interaction compared with the intralayer interaction. As stated in the tight-binding analysis just presented, this region strongly depends on the ratio between the interlayer and intralayer interactions, and monotonically increases up to one third of the $K-\Gamma$ line with increasing interlayer interaction. Indeed, for (111) surfaces of silicon with the diamond structure, the flat-band region is exactly one third of the $K-\Gamma$ line.

Detailed analyses of the electronic energy band near the Fermi level allowed us to unravel the unusual feature of the flat-band states. The flat-band states are split by $10 \mathrm{meV}$ into upper and lower branches with respect to the Fermi level for both majority- and minority-spin states. We thus expect a certain magnetic ordering to appear on the (0001) surfaces of graphite with rhombohedral stacking arrangement. We also noted that the flat-band states were absent in graphite with the Bernal stacking arrangement because its network topology normal to the graphene layer does not satisfy the condition that leads to edge states. ${ }^{8}$

Figure 3 shows the spin density $\left(\Delta \rho=\rho_{\uparrow}-\rho_{\downarrow}\right)$ of a graphite 


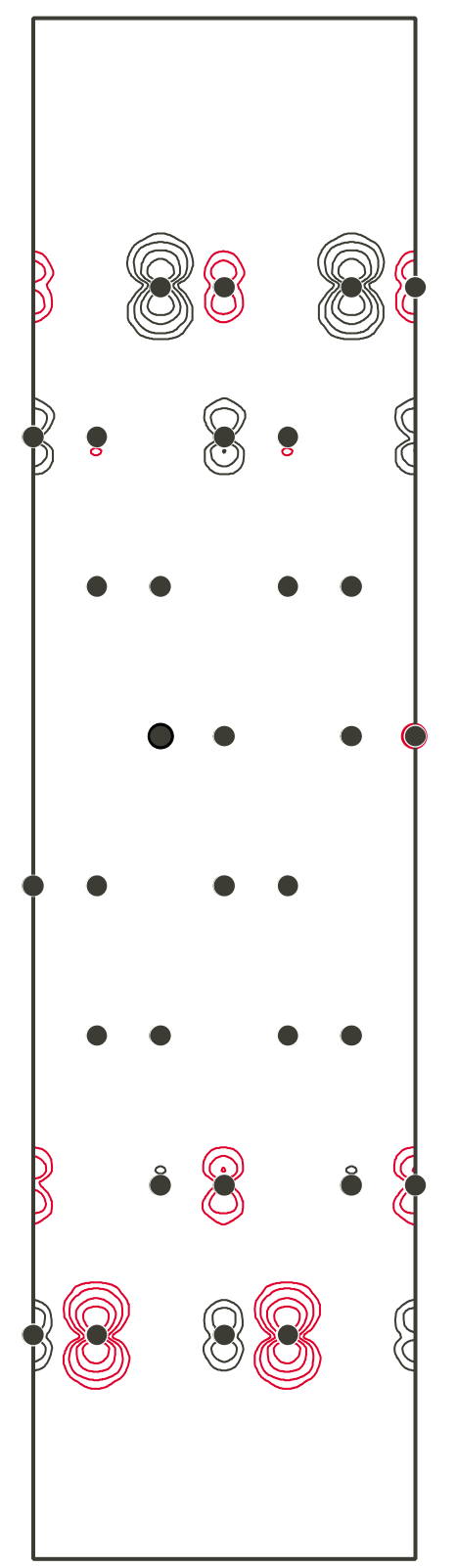

FIG. 3. (Color online) Contour plots of spin density $\Delta \rho=\rho_{\uparrow}(\mathbf{r})$ $-\rho_{\downarrow}(\mathbf{r})$ of rhombohedral graphite. The solid circles denote the position of $\mathrm{C}$ atoms. Positive and negative values of the spin density are shown by black and red lines, respectively. Each contour represents twice (or half) the density of the adjacent contour lines.

slab with a rhombohedral stacking arrangement. The present calculations using local spin density approximation have clearly uncovered the existence of ferrimagnetic spin ordering on the (0001) surfaces, in which the polarized electron spin is mostly distributed on the outermost layers of the graphite slab. Furthermore, the polarized electron spins on the topmost and bottommost layers of the slab are antiparallel so that the net spin on the slab vanishes. There are two atomic sites in rhombohedral graphite and all the atomic sites are classified into the two sublattices $A$ and $B$ (bipartite lattice). On the topmost layer, the polarized electron spin is mainly localized on one of two sublattice (A sublattice), while the spin with opposite direction is mainly localized on (a)

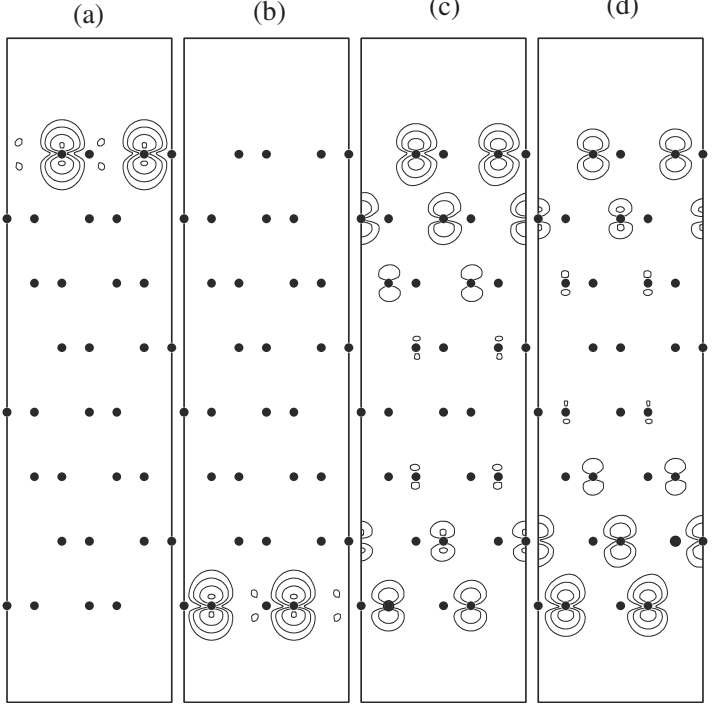

FIG. 4. Contour plots of squared wave functions of rhombohedral graphite at the $K$ point for (a) the lower and (b) higher branches of the flat-band state at the vicinity of the $K$ point along the $K-\Gamma$ line for (c) the lower and (d) higher branches. The solid circles denote the position of $\mathrm{C}$ atoms. Each contour represents twice (or half) the density of the adjacent contour lines.

the $B$ sublattice on the bottommost layer. The distribution of the polarized spin is qualitatively the same as that of the graphene ribbons with zigzag edges resulting from the flat band of edge states. ${ }^{23}$ Thus, based on the analogy with graphene ribbon with a zigzag edge, we consider that the spin polarization on (0001) surfaces of the rhombohedral graphite originates from the edge state on the surfaces.

These results clearly show the possibility of ferrimagnetic spin ordering on the (0001) surfaces of rhombohedral graphite. This spin ordering was localized on one of the two sublattice of the surfaces, though the surfaces did not contain unsaturated covalent bonds, as did the $\mathrm{Si}$ and $\mathrm{Ge}$ surfaces. $^{24,25}$ The calculated amount of the surface polarized spin is $0.036 \mu_{B} / \mathrm{nm}^{2}$. Thus, the (0001) surfaces of rhombohedral graphite are possible candidates for the constituent unit for spintronic devices, as is the case for graphene ribbons with zigzag edges. Perhaps, due to the dimensionality of the surfaces, the magnetic properties are robust against structural and thermal modulations compared with the graphene ribbons.

To elucidate the origin of the surface magnetism, we investigated in detail the wave function associated with the flat-band states. Figures 4(a) and 4(b) show the distribution of the squared wave function of the flat-band states at the $K$ point. Obviously, the state is completely localized at the $K$ point, whose character is inherited from the $\pi$ orbital of the topmost or bottom-most layer of rhombohedral graphite with a nonbonding nature [Figs. 4(a) and 4(b)]. By decreasing the wave number or approaching the $\Gamma$ point, the state loses its surface-localized nature. Indeed, at the vicinity of the $K$ point, the state still exhibits its surface-localized nature but also penetrates inside the slab [Figs. 4(c) and 4(d)]. Then, around the center of the Brillouin zone, the state is distributed throughout the entire graphite slab. The distribution of 
the wave function of the state completely differs from that of a conventional surface-electron state for which the wave function is basically distributed at or near the surface atomic sites throughout the entire Brillouin zone. However, the characteristics are exactly the same as those of the edge state of graphite ribbons with zigzag edges. Thus, the resulting surface state associated with the flat dispersion band is due to a delicate balance of electron transfer among the atoms situated near the surfaces.

Thus, based on both the analytic calculations using the tight-binding model and a first-principles total-energy calculation, we have studied the electronic structure of the (0001) surfaces of rhombohedral graphite. The DFT calculation clearly indicates that the intrinsic magnetic moment emerges on the (0001) surfaces of rhombohedral graphite. The polarized electron spins are ferrimagnetically aligned on the surfaces. We calculate $0.036 \mu_{B} / \mathrm{nm}^{2}$ of surface polarized spin. The spin polarization on the surfaces is ascribed to the flat dispersion band in the vicinity of the $K$ point at the Fermi level. We analyze in detail the wave function associated with the flat-band state to understand the peculiar surfacelocalized nature of the flat-band state: The state localizes on the outermost layer of the slab at the $K$ point. The wave function of the state possesses a $\pi$ character with a nonbonding nature and is distributed on one of two sublattices on the outermost layer. In sharp contrast, with decreasing wave number, the flat-band state loses its localized nature and extends throughout the slab. The characteristics of the wave function and the electronic structure are the same as those of the edge state on graphene ribbons with zigzag edges.

This work was partly supported by CREST, Japan Science and Technology Agency, and a Grant-in-Aid for scientific research from the Ministry of Education, Culture, Sports, Science and Technology of Japan. Computations were performed on an NEC SX-8/4B at the University of Tsukuba, on an NEC SX-8 at the Yukawa Institute of Theoretical Physics, Kyoto University, and on an NEC SX-9 at the Information Synergy Center, Tohoku University.
${ }^{1}$ M. Fujita, K. Wakabayashi, K. Nakada, and K. Kusakabe, J. Phys. Soc. Jpn. 65, 1920 (1996).

${ }^{2}$ K. Nakada, M. Fujita, G. Dresselhaus, and M. S. Dresselhaus, Phys. Rev. B 54, 17954 (1996).

${ }^{3}$ Y. Miyamoto, K. Nakada, and M. Fujita, Phys. Rev. B 59, 9858 (1999).

${ }^{4}$ Y. Shibayama, H. Sato, T. Enoki, and M. Endo, Phys. Rev. Lett. 84, 1744 (2000).

${ }^{5}$ Y. Kobayashi, K.-i. Fukui, T. Enoki, and K. Kusakabe, Phys. Rev. B 73, 125415 (2006).

${ }^{6}$ S. Okada, M. Igami, K. Nakada, and A. Oshiyama, Phys. Rev. B 62, 9896 (2000).

${ }^{7}$ Y. Takagi and S. Okada, Surf. Sci. 602, 2876 (2008).

${ }^{8}$ Y. Takagi, J. Phys. Soc. Jpn. 78, 064701 (2009).

${ }^{9}$ Total energy of the rhombohedral graphite is almost identical to that of the Bernal $(A B)$ graphite in local density approximation calculations.

${ }^{10}$ F. Guinea, A. H. Castro Neto, and N. M. R. Peres, Phys. Rev. B 73, 245426 (2006).
${ }^{11}$ J. L. Manes, F. Guinea, and M. A. H. Vozmediano, Phys. Rev. B 75, 155424 (2007).

${ }^{12}$ M. Koshino and E. McCann, Phys. Rev. B 80, 165409 (2009).

${ }^{13}$ M. Koshino, Phys. Rev. B 81, 125304 (2010).

${ }^{14}$ J. W. McClure, Carbon 7, 425 (1969).

${ }^{15}$ M. S. Dresselhaus and G. Dresselhaus, Adv. Phys. 51, 1 (2002).

${ }^{16}$ P. Hohenberg and W. Kohn, Phys. Rev. 136, B864 (1964).

${ }^{17}$ W. Kohn and L. J. Sham, Phys. Rev. 140, A1133 (1965).

${ }^{18}$ J. P. Perdew and A. Zunger, Phys. Rev. B 23, 5048 (1981).

${ }^{19}$ D. M. Ceperley and B. J. Alder, Phys. Rev. Lett. 45, 566 (1980).

${ }^{20}$ D. Vanderbilt, Phys. Rev. B 41, 7892 (1990).

${ }^{21}$ I. Hamada, M. Otani, O. Sugino, and Y. Morikawa, Phys. Rev. B 80, 165411 (2009).

${ }^{22}$ M. Otani and O. Sugino, Phys. Rev. B 73, 115407 (2006).

${ }^{23}$ S. Okada and A. Oshiyama, Phys. Rev. Lett. 87, 146803 (2001).

${ }^{24}$ J. E. Northrup, J. Ihm, and M. L. Cohen, Phys. Rev. Lett. 47, 1910 (1981).

${ }^{25}$ S. Okada, K. Shiraishi, and A. Oshiyama, Phys. Rev. Lett. 90, 026803 (2003). 